\title{
$\mathbf{N}-\mathbf{O}$ 键解离焓的密度泛函理论研究
}

\author{
郑文锐 ${ }^{*}$ 徐菁利 ${ }^{*}$ 熊 瑞 \\ (上海工程技术大学化学化工学院, 上海 201620)
}

\begin{abstract}
摘要：在高精度计算方法 $\mathrm{G} 3$ 和 G3B3 的基础上, 比较了密度泛函理论(DFT) 十几种方法对 $\mathrm{N}-\mathrm{O}$ 键解离焓 (BDE) 相对于实验值的计算精度, 发现用 B3P86 方法计算 15 种化合物 $\mathrm{N}-\mathrm{O}$ 键的 BDE, 均方根误差最小, 仅为 $6.36 \mathrm{~kJ} \cdot \mathrm{mol}^{-1}$, 计算值与实验值的线性相关系数为 0.991 . 在此基础上, 用该方法分别计算了非芳香化合物及芳香 化合物的 $\mathrm{N}-\mathrm{O}$ 键 BDE. 通过自然键轨道分析, 发现部分 $\mathrm{N}-\mathrm{O}$ 键的 $\mathrm{BDE}$ 与 $\mathrm{N}-\mathrm{O}$ 键的键长、原子电荷密度及 键级之间存在定量关系. 此外, 在 B3P86 方法的基础上预测了几种典型的杂环芳香化合物 $\mathrm{N}-\mathrm{O}$ 键 BDE 值.
\end{abstract}

关键词: 密度泛函理论; 键解离焓; 自然键轨道分析; $\mathrm{N}-\mathrm{O}$ 键 中图分类号: $\mathrm{O} 641$

\section{Density Functional Theory Study on N-O Bond Dissociation Enthalpies}

\author{
ZHENG Wen-Rui * $\quad$ XU Jing-Li ${ }^{*} \quad$ XIONG Rui \\ (College of Chemistry and Chemical Engineering, Shanghai University of Engineering Science, Shanghai 201620, P. R. China)
}

\begin{abstract}
A number of density functional theory (DFT) methods were compared for the calculation of $\mathrm{N}-\mathrm{O}$ bond dissociation enthalpies (BDEs) with the experimental values on the basis of the high-precision calculation methods G3 and G3B3. We found that the B3P86 method gave the lowest root of mean square error of $6.36 \mathrm{~kJ} \cdot \mathrm{mol}^{-1}$ for calculating $\mathrm{N}-\mathrm{O}$ BDE of 15 molecules and the correlation coefficient between the theoretical and experimental values was 0.991. $\mathrm{We}$, therefore, used this method to calculate the $\mathrm{N}-\mathrm{O}$ BDEs of non-aromatic and aromatic compounds. Using natural bond orbital analysis, quantitative relationships between some $\mathrm{N}-\mathrm{O}$ BDEs and the corresponding $\mathrm{N}-\mathrm{O}$ bond lengths, atomic charges, bond orders were determined. In addition, we predicted the $\mathrm{N}-\mathrm{O}$ BDEs of several typical heterocyclic aromatic compounds using the B3P86 method.
\end{abstract}

Key Words : Density functional theory; Bond dissociation enthalpy; Natural bond orbital analysis; $\mathrm{N}-\mathrm{O}$ bond

在化学及生物化学过程中, 总是包含着旧键的 断裂以及新键的生成, 因此需要大量的化学键均裂 解离焓 (BDE) 的热力学数据. 用来测定化合物化学 键解离焓的实验方法较多, 自由基动力学方法、光致 电离质谱分析技术和酸性/电子亲和性循环方法是 目前广泛运用的气相测定方法 ${ }^{[1]}$. 电化学循环和光 声热量测定技术是液相测定键解离焓常用的方法 ${ }^{[2]}$. 但由于实验方法本身的缺陷和自由基寿命较短等原
因导致实验测定键解离焓仍然存在困难, 获得的某 些键解离焓无法验证其可靠性, 目前获得精确的键 解离焓的另一种可行方法是利用量子化学计算 ${ }^{[3]}$.

其中, 从头算方法比一些经验及半经验方法更 受欢迎, 而 Hartree-Fock 及微扰理论由于在处理开 壳层体系时存在自旋污染不适合 BDE 值的计算 ${ }^{[4]}$. 高水平的从头算方法如耦合簇理论方法 (CCSD), 组态相关方法 (QCISD) 计算的 BDE 值比 Hartree-

Received: May 4, 2010; Revised: June 1, 2010; Published on Web: July 22, 2010.

${ }^{*}$ Corresponding authors. ZHENG Wen-Rui, Email: wrzheng@ sues.edu.cn; Tel: +86-21-67791216; Fax: +86-21-67791220.

XU Jing-Li, Email: xujingli@sues.edu.cn; Tel: +86-21-67791220.

The project was supported by the Foundation of Shanghai Excellent Youth (B-8500-08-0110), School Start-Up Fund (08-22) and Innovation Fund (cx0904009), China.

上海优青科研基金(B-8500-08-0110), 校启基金(08-22)和创新项目基金(cx0904009)资助项目

C. Editorial office of Acta Physico-Chimica Sinica 
Fock 及微扰理论方法要好, 但研究表明, 当采用的 基组不够大时, 这些高水平的理论方法仍然大大低 估了 BDE 值 ${ }^{[5}$. 组合从头算方法例如 G3, CBS-Q 方 法虽能成功地用于 BDE 的计算 ${ }^{[4]}$, 但是只适用于处 理含有不超过 8 个非氢原子的体系. 密度泛函理论 (DFT) 方法比组合从头算方法更经济, 且不受自旋 污染的影响, 因此 DFT 方法近年来越来越普遍地运 用到 BDE 的计算中 ${ }^{[6]}$.

$\mathrm{N}-\mathrm{O}$ 键是一类非常重要的键型, 含有 $\mathrm{N}-\mathrm{O}$ 键的化合物在生物医药领域具有重要的研究意义, 例如, 芳香杂环的氮氧化物被认为在临床治疗肿瘤 方面具有一定的作用, 这些化合物本身并不活泼, 然 而在体内酶的作用下能生成自由基中间体并导致细 胞毒素的 DNA 链断裂. 而生成自由基中间体的难 易与 $\mathrm{N}-\mathrm{O}$ 键 BDE 有关 ${ }^{[7]}$. 罗渝然 ${ }^{[8]}$ 搜集了大量的 有机化合物的 $\mathrm{BDE}$ 实验数据, 然而对于 $\mathrm{N}-\mathrm{O}$ 键的 BDE 数据非常有限, 而且有些实验值值得怀疑, 例 如对于 $\mathrm{R}_{3} \mathrm{~N}-\mathrm{O}$ 的 $\mathrm{N}-\mathrm{O}$ 键, 当 $\mathrm{R}$ 为 $\mathrm{CH}_{3}, \mathrm{C}_{2} \mathrm{H}_{5}, n$ $\mathrm{C}_{4} \mathrm{H}_{9}$ 时, 键解离焓的实验值分别为 $(256 \pm 5),(67 \pm 5)$, $(-74 \pm 6) \mathrm{kJ} \cdot \mathrm{mol}^{-1}[$ [9] 取代基 $\mathrm{R}$ 均为烷基, 且烷基链长 相差不大, 然而其 BDE 的实验值却相差很大. 且 $\mathrm{R}$ 为正丁基时, 其 $\mathrm{BDE}$ 数值为负. 对于 $\mathrm{N}-\mathrm{O}$ 键 $\mathrm{BDE}$ 的理论研究, Gomes 等 ${ }^{[7]}$ 运用 B3LYP 方法研究了喹 喔啉- 1,4 二氧化物的 $\mathrm{N}-\mathrm{O}$ 键能的取代基效应, 并 分析了 $\mathrm{N}-\mathrm{O}$ 键 BDE 对一些化合物的抗肿瘤活性 的影响. Gomes 等 ${ }^{[10]}$ 还研究了吩嗪类二氮氧化物衍 生物的 $\mathrm{N}-\mathrm{O}$ 键 BDE, 理论计算采用 B $3 L Y P$ 方法. Ribeiro da Silva 等 ${ }^{[11-12]}$ 结合实验和理论研究, 讨论了 喹喔啉类二氧化物衍生物的热力学性质, 其中包括 $\mathrm{N}-\mathrm{O}$ 键 BDE, 理论计算结果与实验结果具有较好 的一致性.

然而, 对于 $\mathrm{N}-\mathrm{O}$ 键 BDE 的系统的理论研究目 前还尚未见报道. 因此, 本文首先通过比较不同密度 泛函理论方法对 $\mathrm{N}-\mathrm{O}$ 键 $\mathrm{BDE}$ 的计算结果, 找出一 种精度较高的方法, 在此方法的基础上对 $\mathrm{N}-\mathrm{O}$ 键 BDE 进行系统的研究. 不仅可以进一步验证实验数 据的可靠性, 还能从理论角度探讨 $\mathrm{N}-\mathrm{O}$ 键 $\mathrm{BDE}$ 的 影响因素.

\section{1 计算方法}

键均裂解离焓值 BDE 是通过 $101325 \mathrm{~Pa}, 298 \mathrm{~K}$ 气相条件下反应 $\mathrm{X}-\mathrm{Y} \rightarrow \mathrm{X} \cdot+\mathrm{Y} \cdot$ 的焓变值来进行计 算的, 其中反应物分子和生成物自由基在 101325
$\mathrm{Pa}, 298 \mathrm{~K}$ 条件下的焓值由式 $H_{298}=E+\mathrm{ZPE}+H_{\mathrm{trans}}+H_{\mathrm{rot}}+$ $H_{\mathrm{vib}}+R T$ 获得, 其中, $E$ 为电子能量, $Z \mathrm{PE}$ 为零点能, 是对分子电子能量的校正; $H_{\text {trans, }} H_{\text {rot }}, H_{\text {vib }}$ 分别表示 平动、转动和振动对能量的贡献; $R T$ 项为焓值的温 度校正项. 所有分子及自由基在 B3LYP/6-31+G $(d)$ 的水平下进行全优化, 所有构象经过相同水平下的 频率计算以证实为稳定构象, 即所有的振动频率值 为正, 无虚频存在. 分别采用 $\mathrm{B} 3 \mathrm{LYP}^{[13]}, \mathrm{X} 3 \mathrm{LYP}^{[14]}$, $\mathrm{BMK}^{[15]}$, B3P86 ${ }^{[16]}$, MPW1B95 ${ }^{[17]}$, PBE1W ${ }^{[18]}$, TPSS1KCIS $^{[19]}$, MPW1K ${ }^{[20]}$, MPW3LYP ${ }^{[17]}$, TPSSLYPIW ${ }^{[18]}$, MPW1KCIS ${ }^{[2]}$, B2PLYP ${ }^{[2]}$, MPW2PLYP ${ }^{[23]}$, KMLYP ${ }^{[24]}$, MPW1P86 $^{[25]}$, O3LYP ${ }^{[2]}$, MPWLYP1M ${ }^{[27]}$ 十七种 DFT 方法对分子及自由基进行单点能的计算, 综合考虑 基组大小对计算精度的影响以及计算机时, 单点能 的计算基组使用 6-311++G $(2 d f, 2 p)$. 在 B3LYP/6-31+ $\mathrm{G}(d)$ 优化的基础上再用 B3P86/6-31+ $\mathrm{G}(d)$ 方法进行 自然键轨道 $(\mathrm{NBO})$ 分析 ${ }^{[28]}$. 所有计算采用 Gaussian 03 程序包 ${ }^{[29]}$.

\section{2 结果与讨论}

\section{1 各种 DFT 方法的性能评估}

我们选取了 16 个分子的 $\mathrm{N}-\mathrm{O}$ 键 $\mathrm{BDE}$ 的实验 值 ${ }^{[8]}$ 作为参考, 先运用高精度理论方法 $\mathrm{G} 3{ }^{[30]}$ 和 G3B $3^{[31]}$ 来计算其 BDE, 并与实验值进行比较. 但是 由于计算方法的限制, 所选分子的重原子数都不超 过 8. 实验值 ${ }^{[8]}$ 及 G3、G3B3 的计算结果见表 1, 表中 还列出了平均偏差(MD), 平均绝对偏差(MAD)及均 方根误差(RMSE)的值.

从表中可以看出, 除了 $\mathrm{HO}-\mathrm{N}=\mathrm{CHCH}_{3} \mathrm{BDE}$ 的 G3 和 G3B3 计算值均与实验值相差超过了 25 $\mathrm{kJ} \cdot \mathrm{mol}^{-1}$ 外, 其余 15 个 $\mathrm{G} 3$ 和 $\mathrm{G} 3 \mathrm{~B} 3$ 的计算结果与 实验值都吻合得较好, 因此, 分子 $\mathrm{HO}-\mathrm{N}=\mathrm{CHCH}_{3}$ $\mathrm{BDE}$ 的实验值是值得质疑的. 除去这个相差较大的 BDE 值, 从其他 BDE 的 MD, MAD 和 RMSE 值可 以看出, G3B3 方法的均方根误差仅为 $3.83 \mathrm{~kJ} \cdot \mathrm{mol}^{-1}$, 比 $\mathrm{G} 3$ 的结果 $4.42 \mathrm{~kJ} \cdot \mathrm{mol}^{-1}$ 低. 因此 $\mathrm{G} 3 \mathrm{~B} 3$ 的结果 略好于 G3. 其理论值与实验值的线性相关性见图 1. 从图 1 可以看出, 对于 15 个分子的 $\mathrm{N}-\mathrm{O} \mathrm{BDE}$ 的 计算, G3B3 与实验值的线性相关系数为 0.997 .

我们选取了 B3LYP 等十几种 DFT 方法来计算 这 15 个分子的 $\mathrm{N}-\mathrm{O}$ 键 BDE 值, 不同 DFT 方法的 MAD, MD 和 RMSE 列于表 2. 可以看出, B3P86 方 法的MAD 和 RMSE 均最小, 分别为 5.49 和 $6.36 \mathrm{~kJ}$. 
表 116 个 G3、G3B3 计算及实验 $\mathrm{N}-0$ 键解离焓值

Table 1 Experimental and G3, G3B3 calcuated $\mathrm{N}-0$ BDEs for 16 compounds

\begin{tabular}{|c|c|c|c|}
\hline \multirow{2}{*}{ Compound } & \multicolumn{3}{|c|}{$\mathrm{BDE}\left(\mathrm{kJ} \cdot \mathrm{mol}^{-1}\right)$} \\
\hline & G3 & G3B3 & expt. $^{[8]}$ \\
\hline $\mathrm{CH}_{3} \mathrm{O}-\mathrm{NO}$ & 177.1 & 178.7 & 174.9 \\
\hline $\mathrm{C}_{2} \mathrm{H}_{5} \mathrm{O}-\mathrm{NO}$ & 176.4 & 178.1 & 175.7 \\
\hline$n-\mathrm{C}_{3} \mathrm{H}_{7} \mathrm{O}-\mathrm{NO}$ & 176.3 & 178.1 & 179.1 \\
\hline$t-\mathrm{C}_{4} \mathrm{H}_{9} \mathrm{O}-\mathrm{NO}$ & 165.3 & 167.3 & 171.1 \\
\hline $\mathrm{HO}-\mathrm{NO}_{2}$ & 201.7 & 203.4 & 204.2 \\
\hline $\mathrm{CH}_{3} \mathrm{O}-\mathrm{NO}_{2}$ & 178.6 & 178.1 & 174.1 \\
\hline $\mathrm{HOO}-\mathrm{NO}$ & 108.1 & 116.5 & 114.2 \\
\hline$i-\mathrm{C}_{3} \mathrm{H}_{7} \mathrm{O}-\mathrm{NO}$ & 178.7 & 180.1 & 175.3 \\
\hline$n-\mathrm{C}_{4} \mathrm{H}_{9} \mathrm{O}-\mathrm{NO}$ & 176.7 & 178.7 & 177.8 \\
\hline $\mathrm{FO}-\mathrm{NO}_{2}$ & 129.9 & 133.8 & 131.8 \\
\hline $\mathrm{C}_{2} \mathrm{H}_{5} \mathrm{O}-\mathrm{NO}_{2}$ & 180.2 & 179.7 & 172.8 \\
\hline $\mathrm{HOO}-\mathrm{NO}_{2}$ & 98.7 & 101.0 & 100.0 \\
\hline $\mathrm{HO}-\mathrm{N}=\mathrm{CHCH}_{3}$ & 233.8 & 234.3 & 207.9 \\
\hline $\mathrm{CH}_{3} \mathrm{O}-\mathrm{NH}_{2}$ & 231.1 & 230.6 & 228.4 \\
\hline $\mathrm{HO}-\mathrm{NH}_{2}$ & 261.0 & 262.4 & 263.6 \\
\hline $\mathrm{HO}-\mathrm{NHCH}_{3}$ & 261.7 & 262.6 & 271.5 \\
\hline $\mathrm{MD}^{\mathrm{a}}$ & -0.87 & 0.97 & - \\
\hline $\mathrm{MAD}^{\mathrm{a}}$ & 3.65 & 3.07 & - \\
\hline RMSE $^{\mathrm{a}}$ & 4.42 & 3.83 & - \\
\hline
\end{tabular}

MD: mean deviation, MAD: mean absolute deviation, RMSE: root of mean square error; ${ }^{\text {a }}$ The values referred to the results exclusive of $\mathrm{BDE}$ of $\mathrm{HO}-\mathrm{N}=\mathrm{CHCH}_{3}$.

$\mathrm{mol}^{-1}, \mathrm{MD}$ 为 $-2.14 \mathrm{~kJ} \cdot \mathrm{mol}^{-1}$. B3P86 方法指定的是 Becke 三参数混合泛函, 非局域相关由 Perdew 86 提 供. 结果证明对于计算 $\mathrm{N}-\mathrm{O}$ 键 BDE, B3P86 具有 较高的计算精度, 其计算精度的可靠性可能与其本 身的函数形式有关. 使用最多的 B3LYP 泛函方法 的 $\mathrm{RMSE}$ 为 $25.44 \mathrm{~kJ} \cdot \mathrm{mol}^{-1}$, 并且都低估了每一个 $\mathrm{N}-\mathrm{O}$ 键的 BDE 值, 其MD 和 MAD 在数值上相等. BMK 方法是 2004 年由 Boese 和 Martin 发展起来

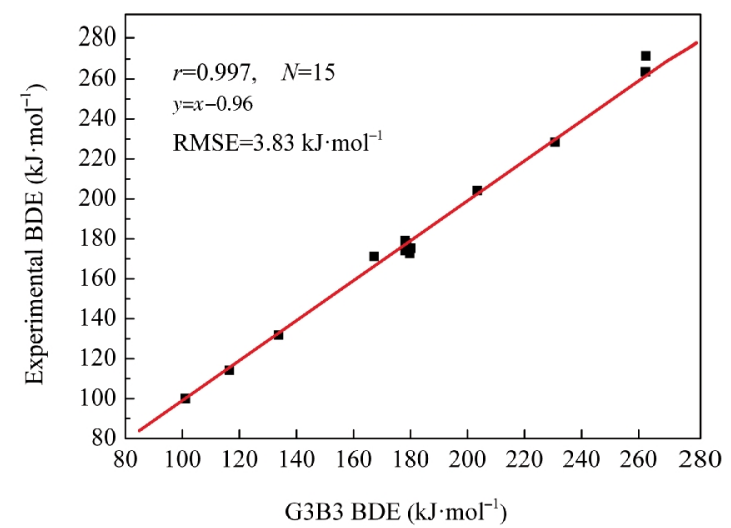

图 1 G3B3 计算与实验的 $\mathrm{N}-\mathrm{O}$ 的 BDE 值之间 的相关关系

Fig.1 Correlation between the experimental and G3B3 calculated $\mathrm{N}-0$ BDEs
表 2 不同密度泛函理论方法计算 BDE 值与实验 BDE 值的 比较

Table 2 Comparison of calculated BDEs by different DFT methods with experimental values

\begin{tabular}{lccc}
\hline DFT method & MAD $\left(\mathrm{kJ} \cdot \mathrm{mol}^{-1}\right)$ & RMSE $\left(\mathrm{kJ} \cdot \mathrm{mol}^{-1}\right)$ & $\mathrm{MD}\left(\mathrm{kJ} \cdot \mathrm{mol}^{-1}\right)$ \\
\hline B3LYP & 24.67 & 25.44 & -24.67 \\
B3P86 & 5.49 & 6.36 & -2.14 \\
ROB3P86 ${ }^{\text {a }}$ & 9.43 & 10.97 & 9.29 \\
BMK & 14.21 & 15.97 & -14.16 \\
MPW1B95 & 11.17 & 12.06 & -9.92 \\
MPW1K & 35.12 & 36.43 & -35.12 \\
MPW3LYP & 18.45 & 19.41 & -18.45 \\
PBE1W & 10.37 & 11.70 & 4.75 \\
TPSS1KCIS & 17.17 & 18.31 & -17.17 \\
TPSSLYPIW & 26.75 & 28.53 & -26.75 \\
MPW1KCIS & 8.38 & 9.94 & -6.84 \\
B2PLYP & 13.15 & 13.65 & -13.15 \\
MPW2PLYP & 15.07 & 15.53 & -15.07 \\
KMLYP & 13.65 & 15.65 & -7.41 \\
MPW1P86 & 8.04 & 9.27 & -7.06 \\
O3LYP & 21.08 & 22.56 & -21.08 \\
X3LYP & 22.90 & 23.68 & -22.90 \\
MPWLYPIM & 11.68 & 14.31 & -11.11 \\
\hline${ }^{a}$ The values referred to the ROB3P86/6-311++G( $(2 d f, 2 p)$ single-point \\
\end{tabular}

的一种新泛函, 对于 $\mathrm{C}-\mathrm{H}$ 键的 $\mathrm{BDE}$ 具有较好的 计算精度 ${ }^{[32]}$, 然而对于 $\mathrm{N}-\mathrm{O}$ 键 BDE 的计算, 其结 果并非最理想. 其中, MPW1K 方法对 $\mathrm{N}-\mathrm{O}$ 键 $\mathrm{BDE}$ 的计算 $\mathrm{RMSE}$ 最大, 为 $36.43 \mathrm{~kJ} \cdot \mathrm{mol}^{-1}$. 此外我 们还采用限制性开壳层方法ROB3P86 对自由基进 行单点能计算, 其 RMSE 为 $10.97 \mathrm{~kJ} \cdot \mathrm{mol}^{-1}$. 因此对 于自由基的单点能计算, 仍是采用默认的(U)B3P86 方法的结果较好. B3P86 方法的计算值与实验值的 线性相关性见图 2 . 从图 2 可以看出, 对于 15 个分

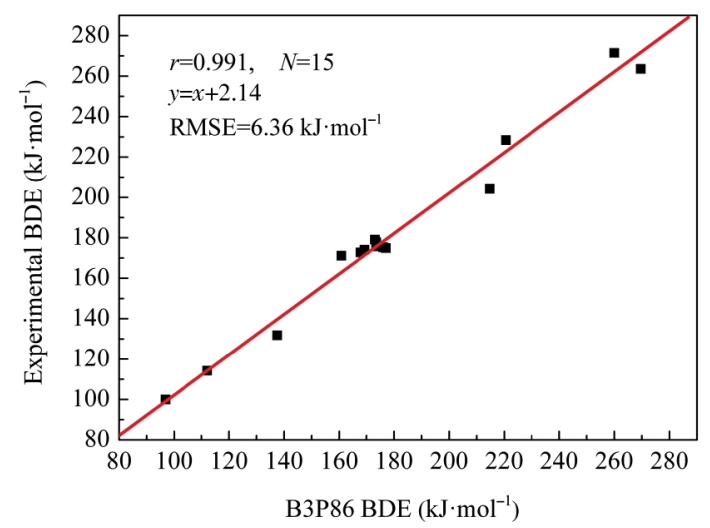

图 2 B3P86 计算与实验的 N-O 的 BDE 值之间 的相关关系

Fig.2 Correlation between the experimental and B3P86 calculated $\mathrm{N}-0$ BDEs 
子的 $\mathrm{N}-\mathrm{O}$ BDE 的计算, B3P86 计算值与实验值的 线性相关系数为 0.991 . 由以上分析, 对以下 $\mathrm{N}-\mathrm{O}$ 键 $\mathrm{BDE}$ 的计算采用 $\mathrm{B} 3 \mathrm{P} 86 / 6-311++\mathrm{G}(2 d f, 2 p) / / \mathrm{B} 3 \mathrm{LYP} /$ $6-31+\mathrm{G}(d)$ 方法.

\section{2 非芳香化合物的 $\mathrm{N}-\mathrm{O}$ 键 BDE}

我们用 B3P86 方法计算了 21 个非芳香类化合 物的 $\mathrm{N}-\mathrm{O}$ 键 $\mathrm{BDE}$, 结果列于表 3. 表中还列出了计 算的 $\mathrm{N}-\mathrm{O}$ 键的键长数据.

从表中可看出, B3P86 计算值与实验值相差 $20 \mathrm{~kJ} \cdot \mathrm{mol}^{-1}$ 以上的化合物有 6 个, 分别为: (1) FO$\mathrm{NO}(\mathrm{No} .1)$, 实验值与计算值相差 $20.1 \mathrm{~kJ} \cdot \mathrm{mol}^{-1}$; (2) $\mathrm{C}_{2} \mathrm{H}_{5} \mathrm{OO}-\mathrm{NO}_{2}(\mathrm{No} .12)$, 实验值与计算值相差 $21.7 \mathrm{~kJ} \cdot$ $\mathrm{mol}^{-1}$; (3) $\mathrm{CH}_{2}=\mathrm{C}\left(\mathrm{CH}_{3}\right) \mathrm{OO}-\mathrm{NO}_{2}(\mathrm{No} .14)$, 实验值与 计算值相差 $33.1 \mathrm{~kJ} \cdot \mathrm{mol}^{-1}$; (4) $\mathrm{C}_{2} \mathrm{H}_{5} \mathrm{COO}-\mathrm{NO}(\mathrm{No} .16)$, 实验值与计算值相差 $33.7 \mathrm{~kJ} \cdot \mathrm{mol}^{-1} ;(5)\left(\mathrm{C}_{2} \mathrm{H}_{5}\right)_{3} \mathrm{~N}-\mathrm{O}$ (No.19), 实验值与计算值相差高达 $184.9 \mathrm{~kJ} \cdot \mathrm{mol}^{-1}$; (6) $\left(n-\mathrm{C}_{4} \mathrm{H}_{9}\right)_{3} \mathrm{~N}-\mathrm{O}(\mathrm{No} .20)$, 实验值与计算值相差高达 $309.4 \mathrm{~kJ} \cdot \mathrm{mol}^{-1}$. 其中, $\left(\mathrm{CH}_{3}\right)_{3} \mathrm{~N}-\mathrm{O},\left(\mathrm{C}_{2} \mathrm{H}_{5}\right)_{3} \mathrm{~N}-\mathrm{O}$ 及 $(n-$ $\left.\mathrm{C}_{4} \mathrm{H}_{9}\right)_{3} \mathrm{~N}-\mathrm{O}$ 属于同一系列化合物, 只是取代烷基 碳链不同, 实验值却相差很大, 分别为 $256.0,67.0$ 及-74.0 kJ· mol ${ }^{-1[-9-9}$. 但是 B3P86 计算值分别为 239.1 , 251.9 及 $235.4 \mathrm{~kJ} \cdot \mathrm{mol}^{-1}$, 彼此相差不大. 从数据看, 我

表 321 个非芳香化合物的 B3P86 计算及实验 $\mathrm{N}-0$ 键 解离焓值

Table 3 Experimental and B3P86 N-O BDEs for 21 non-aromatic compounds

\begin{tabular}{|c|c|c|c|c|}
\hline \multirow{2}{*}{ No. } & \multirow{2}{*}{ Compound } & \multicolumn{2}{|c|}{$\mathrm{BDE}\left(\mathrm{kJ} \cdot \mathrm{mol}^{-1}\right)$} & \multirow{2}{*}{$\begin{array}{l}\mathrm{N}-\mathrm{O} \text { bond } \\
\text { length }(\mathrm{nm})\end{array}$} \\
\hline & & B3P86 & $\operatorname{expt.}^{[8]}$ & \\
\hline 1 & $\mathrm{FO}-\mathrm{NO}$ & 152.6 & 132.5 & 0.15038 \\
\hline 2 & $i-\mathrm{C}_{4} \mathrm{H}_{9} \mathrm{O}-\mathrm{NO}$ & 170.8 & 175.7 & 0.14134 \\
\hline 3 & $s-\mathrm{C}_{4} \mathrm{H}_{9} \mathrm{O}-\mathrm{NO}$ & 176.3 & 173.6 & 0.14077 \\
\hline 4 & $\mathrm{ClO}-\mathrm{NO}_{2}$ & 112.7 & 111.8 & 0.15044 \\
\hline 5 & $\mathrm{C}_{3} \mathrm{H}_{7} \mathrm{O}-\mathrm{NO}_{2}$ & 167.1 & 177.0 & 0.14102 \\
\hline 6 & $i-\mathrm{C}_{3} \mathrm{H}_{7} \mathrm{O}-\mathrm{NO}_{2}$ & 172.7 & 175.7 & 0.14086 \\
\hline 7 & $\mathrm{CH}_{3} \mathrm{OO}-\mathrm{NO}_{2}$ & 90.7 & 86.6 & 0.15100 \\
\hline 8 & $\mathrm{CF}_{3} \mathrm{OO}-\mathrm{NO}_{2}$ & 100.9 & 105.0 & 0.15481 \\
\hline 9 & $\mathrm{CF}_{2} \mathrm{ClOO}-\mathrm{NO}_{2}$ & 102.2 & 106.7 & 0.15379 \\
\hline 10 & $\mathrm{CFCl}_{2} \mathrm{OO}-\mathrm{NO}_{2}$ & 98.8 & 106.7 & 0.15349 \\
\hline 11 & $\mathrm{CCl}_{3} \mathrm{OO}-\mathrm{NO}_{2}$ & 94.9 & 95.8 & 0.15343 \\
\hline 12 & $\mathrm{C}_{2} \mathrm{H}_{5} \mathrm{OO}-\mathrm{NO}_{2}$ & 90.7 & 69.0 & 0.15076 \\
\hline 13 & $\mathrm{CH}_{3} \mathrm{C}(\mathrm{O}) \mathrm{OO}-\mathrm{NO}_{2}$ & 116.4 & 118.8 & 0.15116 \\
\hline 14 & $\mathrm{CH}_{2}=\mathrm{C}\left(\mathrm{CH}_{3}\right) \mathrm{OO}-\mathrm{NO}_{2}$ & 75.7 & 108.8 & 0.14897 \\
\hline 15 & $\mathrm{CH}_{3} \mathrm{COO}-\mathrm{NO}$ & 139.1 & 147.3 & 0.15324 \\
\hline 16 & $\mathrm{C}_{2} \mathrm{H}_{5} \mathrm{COO}-\mathrm{NO}$ & 124.9 & 158.6 & 0.15797 \\
\hline 17 & $\mathrm{CH}\left(\mathrm{CH}_{3}\right)_{2} \mathrm{COO}-\mathrm{NO}$ & 135.1 & 153.6 & 0.15268 \\
\hline 18 & $\left(\mathrm{CH}_{3}\right)_{3} \mathrm{~N}-\mathrm{O}$ & 239.1 & 256.0 & 0.13706 \\
\hline 19 & $\left(\mathrm{C}_{2} \mathrm{H}_{5}\right)_{3} \mathrm{~N}-\mathrm{O}$ & 251.9 & 67.0 & 0.13697 \\
\hline 20 & $\left(n-\mathrm{C}_{4} \mathrm{H}_{9}\right)_{3} \mathrm{~N}-\mathrm{O}$ & 235.4 & -74.0 & 0.13687 \\
\hline 21 & $t-\mathrm{BuN}(\mathrm{O})=\mathrm{N}(\mathrm{O}) t-\mathrm{Bu}$ & 265.0 & 275.1 & 0.12742 \\
\hline
\end{tabular}

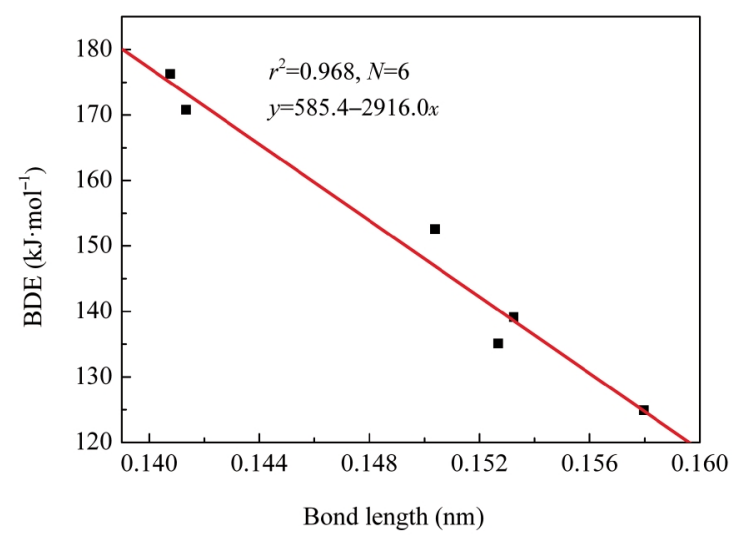

图 3 表 3 中 $\mathrm{O}$-NO 型分子 $\mathrm{N}-\mathrm{O}$ 键 $\mathrm{BDE}$ 与 $\mathrm{N}-\mathrm{O}$ 键长 的相关关系

Fig.3 Relationship between the $\mathrm{N}-\mathrm{O}$ BDEs and $\mathrm{N}-\mathrm{O}$ bond length of $\mathrm{O}-\mathrm{NO}$ molecules in Table 3

们认为计算值更为合理, 因此对 $\left(\mathrm{C}_{2} \mathrm{H}_{5}\right)_{3} \mathrm{~N}-\mathrm{O}$ 与 $(n-$ $\left.\mathrm{C}_{4} \mathrm{H}_{9}\right)_{3} \mathrm{~N}-\mathrm{O}$ 的实验 $\mathrm{N}-\mathrm{O}$ 键 $\mathrm{BDE}$ 值提出质疑. 这两 种物质 $\mathrm{N}-\mathrm{O}$ 键 $\mathrm{BDE}$ 的实验数据, 文献中采用的是 量热法 ${ }^{[9]}$, 其中 $\left(n-\mathrm{C}_{4} \mathrm{H}_{9}\right)_{3} \mathrm{~N}-\mathrm{O}$ 的实验 $\mathrm{N}-\mathrm{O}$ 键 $\mathrm{BDE}$ 值为负值, Acree 等 ${ }^{[33]}$ 认为键解离焓值不可能为负, 因此该实验数据不能被接受.

表 3 中的 $\mathrm{N}-\mathrm{O}$ 键主要包括三种类型: $\mathrm{O}-\mathrm{NO}$ 型, $\mathrm{O}-\mathrm{NO}_{2}$ 型及具有双键性质的 $\mathrm{N}-\mathrm{O}$ 型. 对于其 中 6 个 $\mathrm{O}-\mathrm{NO}$ 型 $\mathrm{N}-\mathrm{O}$ 键, $\mathrm{N}-\mathrm{O}$ 单键的键长与 BDE 计算值之间存在线性关系, 其线性相关系数的 平方值即 $r^{2}$ 为 0.968. 如图 3 所示. 而对于 $\mathrm{O}-\mathrm{NO}_{2}$ 型及具有双键性质的 $\mathrm{N}-\mathrm{O}$ 型的 $\mathrm{N}-\mathrm{O}$ 键 $\mathrm{BDE}$ 计 算值与 $\mathrm{N}-\mathrm{O}$ 键键长之间不存在明显的线性关系.

对于同一系列的化合物, 如 $i-\mathrm{C}_{4} \mathrm{H}_{9} \mathrm{O}-\mathrm{NO}$ 和 $s-$ $\mathrm{C}_{4} \mathrm{H}_{9} \mathrm{O}-\mathrm{NO}, \mathrm{N}-\mathrm{O}$ 键 BDE 的计算值相差不大, 分 别为 170.8 及 $176.3 \mathrm{~kJ} \cdot \mathrm{mol}^{-1}$. 即当氧端取代基为烷 基时, 烷基的改变对 $\mathrm{N}-\mathrm{O}$ 键 $\mathrm{BDE}$ 的影响不大, 类 似的还有 $\mathrm{C}_{3} \mathrm{H}_{7} \mathrm{O}-\mathrm{NO}_{2}$ 和 $i-\mathrm{C}_{3} \mathrm{H}_{7} \mathrm{O}-\mathrm{NO}_{2}, \mathrm{BDE}$ 的计 算值也相差不大. 表中还能看出不同的吸电子取代 基和给电子取代基对 $\mathrm{N}-\mathrm{O}$ 键 $\mathrm{BDE}$ 的影响情况, 例 如 $\mathrm{CH}_{3} \mathrm{OO}-\mathrm{NO}_{2}$ 和 $\mathrm{CF}_{3} \mathrm{OO}-\mathrm{NO}_{2}, \mathrm{BDE}$ 的计算值分 别为 90.7 及 $100.9 \mathrm{~kJ} \cdot \mathrm{mol}^{-1}$, 这是由于 $\mathrm{CH}_{3}$ 为给电子 取代基, $\mathrm{CF}_{3}$ 为吸电子取代基, 因此 $\mathrm{N}-\mathrm{O}$ 键断裂所 生成的相应自由基 $\mathrm{CH}_{3} \mathrm{OO}$ - 比 $\mathrm{CF}_{3} \mathrm{OO}$-更稳定, 所以 对应的分子 $\mathrm{CH}_{3} \mathrm{OO}-\mathrm{NO}_{2}$ 中的 $\mathrm{N}-\mathrm{O}$ 键的 $\mathrm{BDE}$ 小 于 $\mathrm{CF}_{3} \mathrm{OO}-\mathrm{NO}_{2}$.

\section{3 芳香化合物的 $\mathrm{N}-\mathrm{O}$ 键 $\mathrm{BDE}$}

我们用 B3P86 方法计算了 19 个芳香化合物的 $\mathrm{N}-\mathrm{O}$ 键 $\mathrm{BDE}$, 结果列于表 4. 
从表中可以看出, 对于这 19 种芳香化合物 $\mathrm{N}$ $\mathrm{O}$ 键 BDE 的计算, 有 1 个化合物(No.13)实验值与 B3P86 计算值的偏差较大, 为 $20 \mathrm{~kJ} \cdot \mathrm{mol}^{-1}$, 其余化合 物的计算值与实验值偏差均在 $20 \mathrm{~kJ} \cdot \mathrm{mol}^{-1}$ 以内. 例 如, No.5, B3P86计算值 $\left(290.8 \mathrm{~kJ} \cdot \mathrm{mol}^{-1}\right)$ 与实验值 $\left(301.7 \mathrm{~kJ} \cdot \mathrm{mol}^{-1}\right)$ 较为接近; No.6, B3P86 计算值 $(287.8$ $\left.\mathrm{kJ} \cdot \mathrm{mol}^{-1}\right)$ 与实验值 $\left(274.7 \mathrm{~kJ} \cdot \mathrm{mol}^{-1}\right)$ 较为接近; $\mathrm{No}$. 19 , B3P86 计算值 $\left(287.4 \mathrm{~kJ} \cdot \mathrm{mol}^{-1}\right)$ 与实验值 $(294.8$ $\mathrm{kJ} \cdot \mathrm{mol}^{-1}$ )较为接近. 其中, 有些化合物的实验 BDE 被 重复测量过, 不同的实验值之间本身偏差也较大.

表 419 个芳香化合物的 B3P86 计算及实验 $\mathrm{N}-0$ 键解离焓值

Table 4 B3P86 calculated and experimental N-O BDEs for 19 aromatic compounds

\begin{tabular}{|c|c|c|c|c|c|c|}
\hline \multirow{2}{*}{ No. } & \multirow{2}{*}{ Compound } & \multicolumn{2}{|c|}{$\operatorname{BDE}\left(\mathrm{kJ} \cdot \mathrm{mol}^{-1}\right)$} & \multirow{2}{*}{$q_{\mathrm{N}} / e$} & \multirow{2}{*}{$q_{\mathrm{o}} / e$} & \multirow{2}{*}{ WBI } \\
\hline & & B3P86 & expt. ${ }^{[8]}$ & & & \\
\hline 1 & & 139.6 & 141.8 & 0.42453 & -0.4906 & 0.8609 \\
\hline 2 & & 139.1 & 137 & 0.42162 & -0.48819 & 0.8713 \\
\hline 3 & & 138.9 & 136 & 0.41891 & -0.48673 & 0.8802 \\
\hline 4 & & 143.8 & 161.5 & 0.43977 & -0.50424 & 0.8098 \\
\hline 5 & & 290.8 & $\begin{array}{l}\text { (1) } 301.7 \\
\text { (2) } 264.9\end{array}$ & 0.06666 & -0.53825 & 1.2782 \\
\hline 6 & & 287.8 & $\begin{array}{l}\text { (1) } 274.7 \\
\text { (2) } 262.4\end{array}$ & 0.06117 & -0.54847 & 1.2676 \\
\hline 7 & & 259.8 & 278.8 & 0.03164 & -0.53121 & 1.2617 \\
\hline 8 & & 266.7 & 275.9 & 0.09522 & -0.47703 & 1.3243 \\
\hline 9 & & 279.5 & 267.8 & 0.07281 & -0.50854 & 1.2975 \\
\hline 10 & & 287.6 & 300.3 & 0.06981 & -0.52319 & 1.2876 \\
\hline 11 & $\xi 0$ & 295.6 & 288.6 & 0.07765 & -0.51093 & 1.3001 \\
\hline 12 & (O)Ph $\mathrm{Ph}$ & 244.3 & 262.8 & 0.23239 & -0.49917 & 1.2905 \\
\hline 13 & & 303.1 & 283.1 & 0.07931 & -0.54665 & 1.2862 \\
\hline 14 & $\begin{aligned} & \mathrm{O} \\
& \text { stur } \\
&= \mathrm{N}-t-\mathrm{Bu}\end{aligned}$ & 303.7 & 287.6 & 0.09181 & -0.51277 & 1.3135 \\
\hline 15 & $\begin{aligned} & \mathrm{O} \\
= & \mathrm{N}-t-\mathrm{Bu}\end{aligned}$ & 295.4 & 286.0 & 0.07566 & -0.52793 & 1.2890 \\
\hline 16 & & 284.8 & 264.9 & 0.06618 & -0.5201 & 1.2673 \\
\hline 17 & & 281.1 & 267.5 & 0.0574 & -0.53211 & 1.2544 \\
\hline 18 & & 283.7 & 267.3 & 0.06287 & -0.5245 & 1.2625 \\
\hline 19 & & 287.4 & $\begin{array}{l}\text { (1) } 294.8 \\
\text { (2) } 238.9\end{array}$ & 0.07745 & -0.52752 & 1.2731 \\
\hline
\end{tabular}

Waved line in the structures of the compounds indicate the position of bond dissociation; WBI: Wiberg bond index; (1) and (2) in the fourth column indicate different experimetal BDEs. 


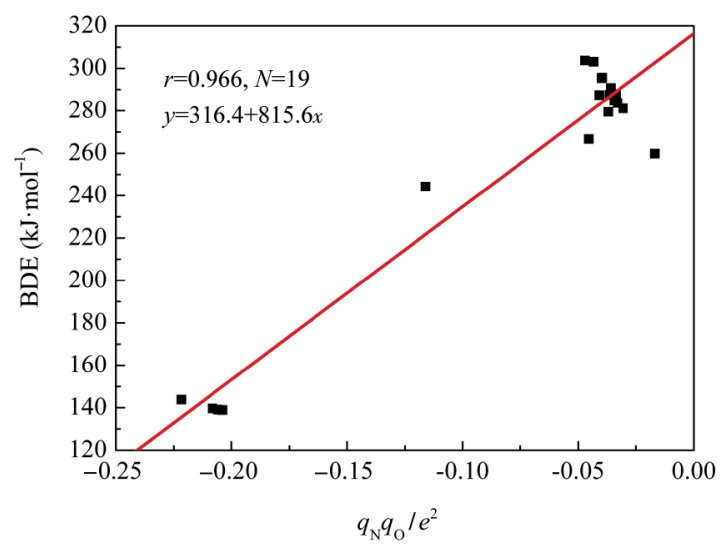

图 4 电荷乘积 $\mathrm{N}-\mathrm{O}$ 键 $\mathrm{BDE}$ 与 $\boldsymbol{q}_{\mathrm{N}} \boldsymbol{q}_{\mathrm{o}}$ 的相关关系

Fig.4 Relationship between the $\mathrm{N}-\mathrm{O}$ BDEs and $q_{\mathrm{N}} q_{0}$

苯环上的远程取代基对 $\mathrm{N}-\mathrm{O}$ 键的影响不显 著, 如 No.1-No.4, 当苯环上对位取代基为吸电子和 给电子取代基时, 吸电子取代基(如硝基)对应的 BDE 较给电子取代基(如甲基)的大, 但差别不大, 不超过 $5 \mathrm{~kJ} \cdot \mathrm{mol}^{-1}$. 同样的规律在 No.13-No.15 及 No.16No.19 中也得到体现, 苯环上邻位和对位取代基的 吸电子及给电子效应对 $\mathrm{BDE}$ 的影响均不大, 但是 $\alpha$ 取代基效应较为明显, 如 No.13 与 No.16, N 原子 $\alpha$ 位为正丁基和苯基时, BDE 值差别较大, 正丁基取 代的较苯基取代的 BDE 大, 此处体现了苯基取代基 较强的给电子共轭效应, 即对应的自由基稳定性较 大导致 BDE 较小. 在所有的氮氧化物中(No.5-No. 19), No.12 的BDE 值最小, 同样也体现了较大的 $\alpha$ 取代基效应.

经过 $\mathrm{NBO}$ 分析, 表中同时列出了 $\mathrm{N}-\mathrm{O}$ 键 $\mathrm{N}$ 原子和 $\mathrm{O}$ 原子的自然电荷密度及以 Wiberg 键级表 示的 $\mathrm{N}-\mathrm{O}$ 键键级, 即 $\mathrm{WBI}$ (Wiberg bond index) ${ }^{[34]}$. 从键级可以看出, No.1-No.4 的 WBI 值在 0.8 左右, 为单键, No.5-No.19 的 WBI 均大于 1, 在 1.2-1.4 之 间, 具有部分双键性质. 此外, 我们发现, 对于表 4 中 所有 $\mathrm{N}-\mathrm{O}$ 键 BDE 的 B3P86 理论值, 不仅与 $\mathrm{N}-\mathrm{O}$ 键氮原子和氧原子的自然电荷密度的乘积呈现线性 关系, 相关系数为 0.966 ; 而且也与 WBI 值呈现线性 关系, 相关系数为 0.967 , 线性相关图分别见图 4 和 图 5.

表 4 中的化合物可分为两类, 一类为 No.1-No. 4 , 断裂的 $\mathrm{N}-\mathrm{O}$ 键为单键, 此外, 氮原子还以双键与 氧原子结合, 氮原子 $2 s$ 轨道的两个电子未参与成 键, 其 BDE 计算值的范围在 $138.9-143.8 \mathrm{~kJ} \cdot \mathrm{mol}^{-1}$ 之间; 另一类为氮氧化物, 为 No.5-No.19, 断裂的 $\mathrm{N}-\mathrm{O}$ 键具有双键特征, 氮原子 $2 s$ 轨道的两个电子

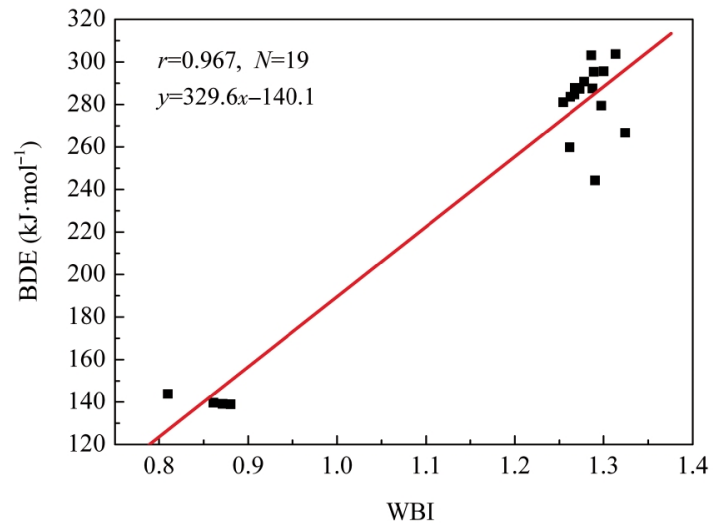

图 $5 \mathrm{~N}-\mathrm{O}$ 键 BDE 与 $\mathrm{N}-\mathrm{O}$ 键键级的相关关系 Fig.5 Relationship between BDEs and WBI of $\mathrm{N}-\mathrm{O}$

参与成键, 分子中形成了不定域 $\pi$ 键, 其 $\mathrm{BDE}$ 计算 值的范围在 $244.3-303.7 \mathrm{~kJ} \cdot \mathrm{mol}^{-1}$ 之间, 远远大于第 一类化合物. 可以看出, $\mathrm{N}-\mathrm{O}$ 键的不同成键特征对 其 BDE 有较大的影响, 离域 $\pi$ 键的形成使得 $\mathrm{N}-\mathrm{O}$ 键较难断裂, BDE 值较大.

\section{4 几种典型芳香杂环氮氧化物的 $\mathrm{N}-0$ 键 BDE 预测}

由于芳香杂环的氮氧化物被认为在临床治疗肿 瘤方面具有一定的作用, 其 $\mathrm{N}-\mathrm{O}$ 键的 BDE 对研究 作用机理有一定的指导意义, 我们用 B3P86 方法预

\section{表 59 种典型芳香杂环氮氧化物 $\mathrm{N}-\mathrm{O}$ 键 BDE 的 B3P86 理论预测值}

Table 5 B3P86 theoretical prediction values of $\mathbf{N}-0$ BDE for 9 typical heteroaromatic nitrogen oxides

\begin{tabular}{ll}
\hline No. & BDE $\left(\mathrm{kJ} \cdot \mathrm{mol}^{-1}\right)$ \\
\hline & \\
\hline
\end{tabular}


测了几种典型芳香杂环氮氧化物的 $\mathrm{N}-\mathrm{O}$ 键BDE, 结果列于表 5 .

从表中可以看出, 含有 2 个氮原子的六元氮杂 环其氮氧化物的 $\mathrm{N}-\mathrm{O}$ 键 BDE 值与环上两个氮原 子的相对位置有关, 即 No.1-No.3, 其 BDE 值大小 顺序为哒嗪>吡嗪>嘧啶, 即两个氮原子处于邻位时, 其 BDE 值最大, 处于间位时, 其 BDE 最小, 最大值 与最小值之间相差 $25 \mathrm{~kJ} \cdot \mathrm{mol}^{-1}$ 以上. No.4 及 No. 5 的喹啉氮氧化物和异喹啉氮氧化物的 BDE 值差别 不大, 说明其 BDE 值受氮原子在环上的位置影响不 大. 这一规律也在嘌呤环上得到证实, 即 No.6-No.8, 六元环上的 $\mathrm{N}$ 原子位置不影响其 $\mathrm{N}-\mathrm{O}$ 键 $\mathrm{BDE}$, 而 五元环位置上的氮原子其 $\mathrm{N}-\mathrm{O}$ 键 $\mathrm{BDE}$ 与六元环 位置的 $\mathrm{BDE}$ 相差 $25 \mathrm{~kJ} \cdot \mathrm{mol}^{-1}$ 以上, 说明环的类型 对 BDE 的影响较大. 呋啶环氮氧化物(No.9)的 BDE 与喹啉环氮氧化物(No.4)的 BDE 相差不大, 也体现 了远程取代基效应不明显的规律.

\section{3 结 论}

在高精度计算方法 G3 和 G3B3 的基础上, 比 较了十几种 DFT 方法对 $\mathrm{N}-\mathrm{O}$ 键 BDE 的计算精 度, 发现 B3P86 方法对于 15 种化合物 $\mathrm{N}-\mathrm{O}$ 键 $\mathrm{BDE}$ 的计算, 均方根误差最小, 为 $6.36 \mathrm{~kJ} \cdot \mathrm{mol}^{-1}$, 计 算值与实验值的线性相关系数为 0.991 . 在此基础 上, 用该方法分别计算了非芳香化合物及芳香化合 物的 $\mathrm{N}-\mathrm{O}$ 键 $\mathrm{BDE}$, 对某些实验值提出了质疑, 计 算结果还体现了不明显的远程取代基效应及较明显 的 $\alpha$ 取代基效应，同时通过自然键轨道分析, 发现 部分 $\mathrm{N}-\mathrm{O}$ 键的 $\mathrm{BDE}$ 与 $\mathrm{N}-\mathrm{O}$ 键的键长、原子电荷 密度及键级之间均存在定量关系. 此外, 鉴于芳香杂 环的氮氧化物在临床治疗肿瘤方面的重要作用, 在 B3P86 方法的基础上预测了几种典型的杂环芳香化 合物 $\mathrm{N}-\mathrm{O}$ 键 $\mathrm{BDE}$ 值.

\section{References}

1 (a) Berkowitz, J.; Ellison, G. B.; Gutman, D. J. Phys. Chem., 1994, 98: 2744

(b) Zhu, X. Q.; Li, H. R.; Ai, T.; Lu, J. Y.; Yang, Y.; Cheng, J. P. Chem.-Eur. J., 2003, 9: 871

2 (a) Bordwell, F. H.; Zhang, X. M. Acc. Chem. Res., 1993, 26: 510 (b) Larhoven, L. J. J.; Mulder, P.; Wayner, D. D. M. Acc. Chem. Res., 1999, 32: 342

3 (a) Sun, Y. M.; Zhang, H. Y.; Chen, D. Z.; Liu, C. B. Org. Lett., 2002, 4: 2909 (b) Wang, L. F.; Zhang, H. Y. Bioorg. Med. Chem. Lett., 2003, 13: 3789

(c) Yao, X. Q.; Hou, X. J.; Jiao, H.; Xiang, H. M.; Li, Y. W.

J. Phys. Chem. A, 2003, 107: 9991

(d) Turecek, F. J. Am. Chem. Soc., 2003, 125: 5954

(e) Shen, L.; Zhang, H. Y.; Ji, H. F. Org. Lett., 2005, 7: 243

4 (a) Feng, Y.; Liu, L.; Wang, J. T.; Huang, H.; Guo, Q. X. J. Chem. Inf. Comput. Sci., 2003, 43: 2005

(b) Qi, X. J.; Li, Z.; Fu, Y.; Guo, Q. X.; Liu, L. Organometallics, 2008, 27: 2688

5 (a) Henry, D. J.; Parkinson, C. J.; Mayer, P. M.; Radom, L. J. Phys. Chem. A, 2001, 105: 6750

(b) Song, K. S.; Cheng, Y. H.; Fu, Y.; Liu, L.; Li, X. S.; Guo, Q. X. J. Phys. Chem. A, 2002, 106: 6651

6 (a) Pratt, D. A.; Mills, J. H.; Porter, N. A. J. Am. Chem. Soc., 2003, 125: 5801

(b) Zhang, H. Y.; Sun, Y. M.; Wang, X. L. Chem. Eur. J., 2003, 9: 502

(c) Lin, C. L.; Lai, C. H.; Chu, S. Y. Chem. Phys. Lett., 2002, 359: 355

(d) Zhang, H. Y.; Sun, Y. M.; Chen, D. Z. Chin. Chem. Lett., 2002, 13: 531

(e) Yao, X. Q.; Hou, X. J.; Wu, G. S.; Xu, Y. Y.; Xiang, H. W.; Jiao, H.; Li, Y. W. J. Phys. Chem. A, 2002, 106: 7184

(f) Lue, J. M.; Wittbrodt, J. M.; Wang, K.; Wen, Z.; Schlegel, H. B.; Wang, P. G.; Cheng, J. P. J. Am. Chem. Soc., 2001, 123: 2903 (g) Feng, Y.; Liu, L.; Wang, J. T.; Zhao, S. W.; Guo, Q. X. J. Org Chem., 2004, 69: 3129

7 Gomes, J. R. B.; Ribeiro da Silva, M. D. M. C.; Ribeiro da Silva, M. A. V. Chem. Phys. Lett., 2006, 429: 18

8 Luo, Y. R. Handbook of bond dissociation energies. Beijing: Science Press, 2005: 212-219［罗渝然. 化学键能数据手册. 北 京: 科学出版社, 2005: 212-219]

9 Acree Jr.,W. E.; Tucker, S. A.; Ribeiro da Silva, M. D. M. C.; Matos, M. A. R.; Goncalves, J. M.; Ribeiro da Silva, M. A. V.; Pilcher, G. J. Chem. Thermody., 1995, 27: 391

10 Gomes, J. R. B.; Sousa, E. A.; Goncalves, J. M.; Monte, M. J. S.; Gomes, P.; Pandey, S.; Acree Jr.,W. E.; Ribeiro da Silva, M. D. M. C. J. Phys. Chem. B, 2005, 109: 16188

11 Ribeiro da Silva, M. D. M. C.; Gomes, J. R. B.; Goncalves, J. M.; Sousa, E. A.; Pandey, S.; Acree Jr., W. E. Org. Biomol. Chem., 2004, 2: 2507

12 Ribeiro da Silva, M. D. M. C.; Gomes, J. R. B.; Goncalves, J. M.; Sousa, E. A.; Pandey, S.; Acree Jr., W. E. J. Org. Chem., 2004, 69 2785

13 (a) Becke, A. D. Phys. Rev. A, 1988, 38: 3098 (b) Lee, C.; Yang, W.; Parr, R. G. Phys. Rev. B, 1988, 37: 785

14 Xu, X.; Goddard, W. A. Proc. Natl. Acad. Sci. U. S. A., 2004, 101 2673

15 Boese, A. D.; Martin, J. M. L. J. Chem. Phys., 2004, 121: 3405

16 Perdew, J. P. Phys. Rev. B, 1986, 33: 8822 
17 Zhao, Y.; Truhlar, D. G. J. Phys. Chem. A, 2004, 108: 6908

18 Dahlke, E. E.; Truhlar, D. G. J. Phys. Chem. B, 2005, 109: 15677

19 Zhao, Y.; Truhlar, D. G. Phys. Chem. Chem. Phys., 2005, 7: 2701

20 Lynch, B. J.; Zhao, Y.; Truhlar, D. G. J. Phys. Chem. A, 2003, 107: 1384

21 Zhao, Y.; Gonzalez-Garcia, N.; Truhlar, D. G. J. Phys. Chem. A, 2005, 109: 2012

22 Grimme, S. J. Chem. Phys., 2006, 124: 10342

23 Schwabe, T.; Grimme, S. Phys. Chem. Chem. Phys., 2006, 8: 4398

24 Kang, J. K.; Musgrave, C. B. J. Chem. Phys., 2001, 115: 11040

25 Perdew, J. P.; Burke, K.; Wang, Y. Phys. Rev. B, 1996, 54: 16533

26 Hoe, W. M.; Cohen, A. J.; Handy, N. C. Chem. Phys. Lett., 2001, 341: 319

27 Schultz-Fademrecht, C.; Tius, M. A.; Grimme, S.; Wibbeling, B.; Hoppe, D. Angew. Chem. Int. Edit., 2002, 41: 1532

28 (a) Reed, A. E.; Curtiss, L. A.; Weinhold, F. Chem. Rev., 1988, 88:
899

(b) Cheng, Y. H.; Zhao, X.; Song, K. S.; Liu, L.; Guo, Q. X. J. Org. Chem., 2002, 67: 6638

29 Frisch, M. J.; Trucks, G. W.; Schlegel, H. B.; et al. Gaussian 03. Revision A.01. Pittsburgh, PA: Gaussian Inc., 2003

30 Curtiss, L. A.; Raghavachari, K.; Redfern, P. C.; Rassolov, V.; Pople, J. A. J. Chem. Phys., 1998, 109: 7764

31 Baboul, A. G.; Curtiss, L. A.; Redfern, P. C.; Raghavachari, K. J. Chem. Phys., 1999, 110: 7650

32 Zheng, W. R.; Fu, Y.; Wang, H. J.; Guo, Q. X. Chin. J. Org. Chem., 2008, 28: 459 [郑文锐, 傅 尧, 王华静, 郭庆祥. 有机化学, 2008, 28: 459]

33 Acree Jr., W. E.; Pilcher, G.; Ribeiro da Silva, M. D. M. C. J. Phys. Chem. Ref. Data, 2005, 34: 553

34 Glendening, E. D.; Weinhold, F. J. Comput. Chem., 1998, 19: 610 